\title{
Influence of Diverse Atmospheric Conditions on Optical Properties of a Pulse Laser in a Time-of-Flight Laser Range Finder
}

\author{
Young Bo Shim ${ }^{1}$, Oh-Jang Kwon ${ }^{1}$, Hyun-Yong $\mathrm{Choi}^{2}$, and Young-Geun $\mathrm{Han}^{1 \text { * }}$ \\ ${ }^{I}$ Department of Physics and Research Institute for Natural Sciences, \\ Hanyang University, Seoul 133-791, Korea \\ ${ }^{2} I T$ Convergence Center, KETI, Gwangju 500-480, Korea
}

(Received August 5, 2014 : revised September 25, 2014 : accepted September 25, 2014)

\begin{abstract}
We investigate the propagation characteristics of a pulse laser in a time-of-flight laser range finder (TOF-LRF) system with variations in atmospheric conditions, such as temperature, pressure, relative humidity, and the concentration of $\mathrm{CO}_{2}$. The measurement error of distance related with the group velocity change in the TOF-LRF system is analyzed by considering the refractive index of the standard atmosphere with variations in atmospheric conditions. The dependence of the pulse width broadening induced by chromatic dispersion of the standard atmosphere on the operating wavelength and the initial pulse width of the light sources is discussed. The transmission of air with variations in the relative humidity or the concentration of $\mathrm{CO}_{2}$ is analyzed by using different values of absorption coefficients depending on the operation wavelength of the light source in the TOF-LRF system.
\end{abstract}

Keywords : Refractive index change, Time delay, Optical absorption in air, Pulse laser, Laser rangefinder OCIS codes : (010.1300) Atmospheric propagation; (010.1030) Absorption; (260.2030) Dispersion; (280.3400) Laser range finder

\section{INTRODUCTION}

The time-of-flight laser range finder (TOF-LRF) system have been attracted in a variety of applications, such as military, geometrical surveying, robotics, and autonomous vehicles in order to measure distance precisely [1]. In the TOF-LRF system, the distance to the target is determined by measuring round trip time after propagating a pulse laser through air [1]. In the TOF-LRF system, a pulse laser with short pulse width can dramatically improve the measurement resolution of distance [2-5]. Recently, a femtosecond laser was implemented for the precise measurement of distance in the TOF-LRF system to effectively improve the resolution $[6,7]$. For the TOF-LRF system, however, the main limitation of the precise measurement of distance is the unexpected property change of the pulse laser caused by the variation of the standard atmospheric conditions [8]. The measurement error of distance is usually induced by the variation of group velocity because of the pulse width broadening [8-11]. The refractive index of air is analyzed with respect to the atmospheric conditions $[12,13]$. The absorption of air related to the molecules of air constituents is investigated [14-16]. However, it is not enough to analyze the propagation characteristics of the pulse laser traveling in the air including group velocity, pulse width broadening, and transmission. Therefore, it is necessary to analyze the propagation characteristics of the pulse laser propagating though the air to improve the performance of the TOF-LRF system. In this paper, we discuss the effect of atmospheric conditions on variations of optical properties of a pulse laser in the TOF-LRF system, such as the measurement error of distance, the pulse width broadening, and the transmission. The measurement error of distance in the TOF-LRF system caused by the group velocity change is analyzed by calculating the refractive index of the standard atmosphere with variations in atmospheric conditions, such as temperature, pressure, relative humidity, and the concentration of $\mathrm{CO}_{2}$. The variation of the pulse width broadening induced by chromatic dispersion of the standard atmosphere is also analyzed depending on the operating wavelength and the initial pulse width of the light source in the TOFLRF system. The transmission of air with variations in the

\footnotetext{
*Corresponding author: yghan@hanyang.ac.kr

Color versions of one or more of the figures in this paper are available online.
} 
relative humidity or the concentration of $\mathrm{CO}_{2}$ is theoretically analyzed by using different values of absorption coefficients depending on the operation wavelength of the light source in the TOF-LRF system.

\section{RESULTS AND DISCUSSION}

The variation of the refractive index of air with variations in atmospheric conditions, such as temperature, pressure, relative humidity, and the concentration of $\mathrm{CO}_{2}$, can be theoretically calculated. The refractive index of air (n) can be written as [13],

$$
\begin{aligned}
& n=1+\frac{\rho_{a}}{M_{a}}\left[\left(\frac{136897 \times 10^{-8}}{238.0185-\frac{1}{\lambda_{0}{ }^{2}}}+\frac{3968.75 \times 10^{-8}}{57.362-\frac{1}{\lambda_{0}{ }^{2}}}\right)\left(1+5.34 \times 10^{-7}\left(x_{c}-450\right)\right)\right] \\
& +\rho_{w}\left(3.06034 \times 10^{-4}+2.7388 \times 10^{-6} \frac{1}{\lambda_{0}{ }^{2}}-3.3564 \times 10^{-8} \frac{1}{\lambda_{0}{ }^{4}}\right. \\
& \left.+4.175 \times 10^{-9} \frac{1}{\lambda_{0}^{6}}\right), \\
& M_{a}=2.89586956 \times 10^{-2}+12.011 \times 10^{-9} x_{c}, \\
& \rho_{a}=\left(3.48433 \times 10^{-3}+1.4452 \times 10^{-9} x_{c}\right) P \frac{1-x_{w}}{T} \\
& \rho_{w}=0.000216758 P \frac{x_{w}}{T}, \\
& x_{w}=\left(1.00062+3.14 \times 10^{-8} P+5.6 \times 10^{-7} T^{2}\right) \frac{S}{P} h \\
& s=\exp \left[1.237885 \times 10^{-5}(T+273.15)^{2}-1.9121316 \times 10^{-2} T\right. \\
& \left.+39.160098-\frac{6343.1645}{T+273.15}\right],
\end{aligned}
$$

where, $\rho_{a}$ is the mass density of the dry air in the atmosphere, $M_{a}$ is the molar mass of the dry air, $\lambda_{0}$ is the operating wavelength of the light source in the TOF-LRF system, $x_{c}$ is the concentration of $\mathrm{CO}_{2}, \rho_{w}$ is the mass density of water vapor in the atmosphere, $P$ and $T$ are the pressure and temperature, respectively. $x_{w}$ is the molar fraction of the water vapor, $s$ is the saturation vapor pressure, and $h$ is the relative humidity.

Figures 1(a), 1(b), 1(c), and 1(d) show theoretical results for the refractive index change of air with variations in atmospheric conditions, such as temperature, pressure, humidity, and the concentration of $\mathrm{CO}_{2}$, respectively. To calculate the dependence of the refractive index of air on temperature, three parameters (pressure, humidity, and the concentration of $\mathrm{CO}_{2}$ ) were kept as constant at the standard atmosphere (pressure: $101.33 \mathrm{kPa}$, humidity: $0 \%$, and the concentration of $\mathrm{CO}_{2}: 300 \mathrm{ppm}$ ). The increase of temperature reduces the refractive index of air because the active thermal molecular motion in a certain volume diminishes the density of air as seen in Fig. 1(a) [13]. As temperature is changed from $-15^{\circ} \mathrm{C}$ to $45^{\circ} \mathrm{C}$, the variation of refractive index of air is estimated to be $\sim 5.97 \times 10^{-5}$.

To analyze the effect of pressure on the refractive index of air, temperature, humidity, and the concentration of $\mathrm{CO}_{2}$ were kept constant to be $15^{\circ} \mathrm{C}, 0 \%$, and $300 \mathrm{ppm}$, respectively. By considering the ideal gas equation, we can readily expect that the refractive index of air changes to higher values under higher pressure at constant temperature and volume. As pressure is changed from $80 \mathrm{kPa}$ to 120 $\mathrm{kPa}$, the total amount of refractive index variation of air is approximated to be $\sim 1.12 \times 10^{-4}$ as shown in Fig. 1 (b). By considering the group velocity of air, the target distance $\left(D_{t}\right)$ can be written as [8]

$$
D_{t}=\frac{c_{0}}{2\left(n-\lambda_{0}\left(\frac{d n}{d \lambda}\right)\right)} t_{g},
$$

where $c_{0}$ is the speed of light, and the $t_{g}$ is the travel time of the laser pulse. The dependence of refractive index of air on atmospheric conditions should definitely induce the measurement distance error of the pulse laser in the TOFLRF system. By using all results in Fig. 1(b) and Eq. (7), it is evident that the variation of refractive index induced by temperature and pressure results in maximum measurement errors of distances of $6.42 \mathrm{~mm}$ and $12.01 \mathrm{~mm}$, respectively, for the 200-m round trip distance through air.

Temperature, pressure, and the concentration of $\mathrm{CO}_{2}$ were kept constant to be $15^{\circ} \mathrm{C}, 101.33 \mathrm{kPa}$, and $300 \mathrm{ppm}$, respectively, to calculate the variation of the refractive index of air with respect to humidity. The variation of refractive index of air is calculated to be $6.06 \times 10^{-7}$ as humidity is changed from 0 to $100 \%$ as shown in Fig. 1 (c). Humidity decreases the refractive index of air because water vapor has $15 \%$ lower refractive index than dry air [17]. As the concentration of $\mathrm{CO}_{2}$ is changed from 0 to $3000 \mathrm{ppm}$ for a fixed humidity of $0 \%$, the variation of refractive index of air is calculated to be $4.53 \times 10^{-7}$ as shown in Fig. 1 (d). The concentration of $\mathrm{CO}_{2}$ increases the refractive index of air because the air with $\mathrm{CO}_{2}$ has $50 \%$ higher refractive index than the air without $\mathrm{CO}_{2}$ [18]. The variation of refractive index induced by humidity and the concentration of $\mathrm{CO}_{2}$ results in maximum measurement errors of distances of $56.86 \mu \mathrm{m}$ and $48.98 \mu \mathrm{m}$, respectively, for the $200-\mathrm{m}$ round trip distance through air.

When a laser pulse in a TOF-LRF system propagates through a dispersive medium, the pulse width is broadened 


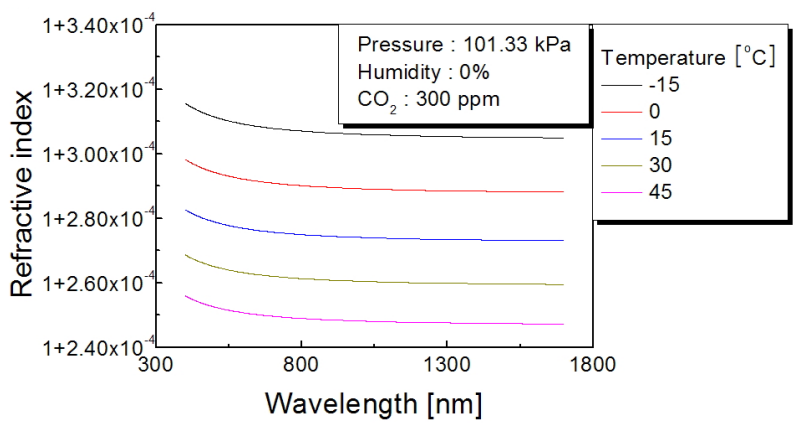

(a)

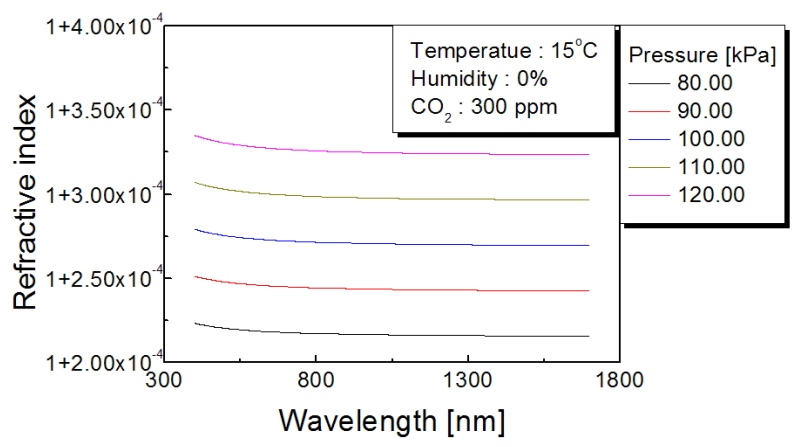

(b)

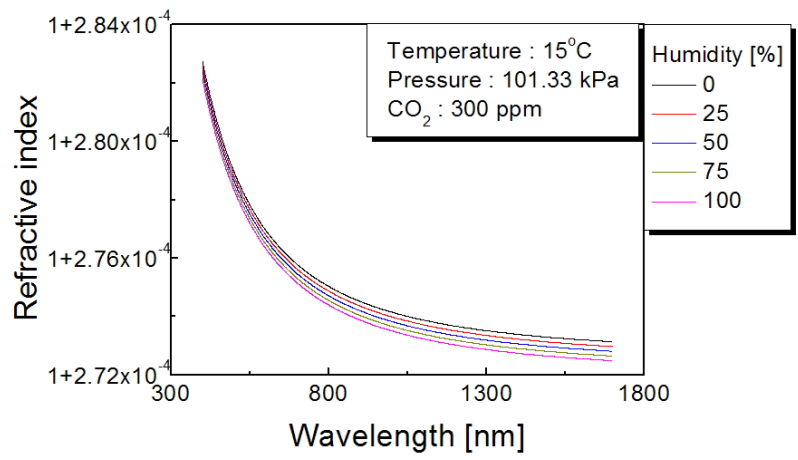

(c)

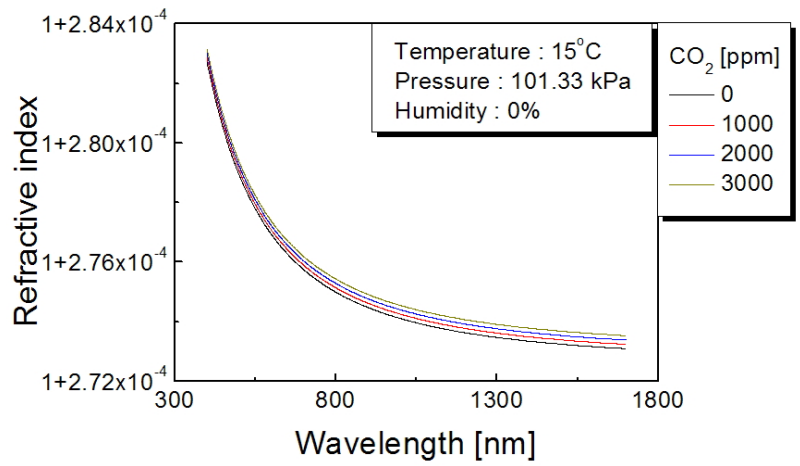

(d)

FIG. 1. Theoretical results for the refractive index change of air with variations in atmospheric conditions: (a) temperature, (b) pressure, (c) humidity, and (d) the concentration of $\mathrm{CO}_{2}$.

because of the chromatic dispersion of the medium. The absolute values of the chromatic dispersion $(|D|)$ can be derived from the refractive index of air as [19],

$$
|D|=\left|-\left(\frac{\lambda_{0}}{c_{0}}\right) \frac{d^{2} n}{d \lambda^{2}}\right|
$$

The theoretical result of the absolute value of the chromatic dispersion of the standard atmosphere is shown in Fig. 2(a). The chromatic dispersion of air with respect to the wavelength is changed in a range from $-5.95 \times 10^{-1}$ to $-5.33 \times 10^{-3} \mathrm{ps} / \mathrm{km} / \mathrm{nm}$. Since the chromatic dispersion depends on the wavelength, the amount of pulse width broadening should be calibrated by considering the operating wavelength of the TOF-LRF system. The amount of the pulse width broadening $(\Delta \tau)$ caused by the chromatic dispersion after the round trip of the pulse laser can be written as [19],

$$
\Delta \tau=2 D_{t} \Delta \lambda|D|
$$

where $\Delta \lambda$ is the spectral width of the pulse laser, which can be expressed by

$$
\Delta \lambda=c_{0}\left(\frac{1}{f_{0}-\Delta f / 2}-\frac{1}{f_{0}+\Delta f / 2}\right)=c_{0}\left(\frac{\Delta f}{f_{0}^{2}-(\Delta f / 2)^{2}}\right),
$$

where $f_{0}=c_{0} / \lambda_{0}$ and $\Delta f$ is the spectral width in the frequency domain. For an unchirped transform-limited Gaussian pulse laser with a time-bandwidth product of 0.44 , the spectral width $(\Delta f)$ in the frequency domain can be written as [20],

$$
\Delta f=\frac{0.44}{\tau_{0}},
$$

where $\tau_{0}$ is the initial pulse width. By substituting Eqs. (10) and (11) into Eq. (9), we obtain the amount of the pulse width broadening $(\Delta \tau)$ as [19],

$$
\begin{aligned}
\Delta \tau & =2 D_{t}|D| \tau_{0} \lambda_{0} c_{0}\left(\frac{1}{\tau_{0} c_{0}-0.22 \lambda_{0}}-\frac{1}{\tau_{0} c_{0}+0.22 \lambda_{0}}\right) \\
& =2 D_{t}|D| \tau_{0} \lambda_{0} c_{0}\left(\frac{0.44 \lambda_{0}}{\left(\tau_{0} c_{0}\right)^{2}-\left(0.22 \lambda_{0}\right)^{2}}\right)
\end{aligned}
$$

Figure 2(b) shows the variation of the amount of pulse width broadening of the pulse laser after the 200-m round trip to the target. The amount of pulse width broadening should be severely changed by the operating wavelength of the input light source because of the chromatic dispersion of the standard atmosphere. By considering Eq. (9), it is evident that the amount of the pulse width broadening $(\Delta \tau)$ is directly proportional to the absolute value of the chromatic 
dispersion of the standard atmosphere. As seen in Fig. 2(a), increasing the operating wavelength of the input light source gradually diminishes the absolute value of the chromatic dispersion of the standard atmosphere. The amount of the pulse width broadening $(\Delta \tau)$ should be substantially reduced by increasing the operating wavelength of the input light source as seen in Fig. 2(b).

The pulse width broadening strongly depends on the initial pulse width of the input light source. Since the spectral width of the pulse laser $(\Delta \lambda)$ is reduced by increasing its initial pulse width $(\tau)$, the amount of the pulse width broadening should be changed by the initial pulse width $\left(\tau_{0}\right)$. For the case of the femtosecond laser, its spectral width is obviously much broader than that of the nanosecond or picosecond laser, the amount of the pulse width broadening for the femtosecond laser should be higher than those of other pulse lasers as seen in Fig. 2(b). The amount of pulse width broadening for various pulse lasers with different initial pulse width were shown in Table 1.

The measurement accuracy of distance in the TOF-LRF

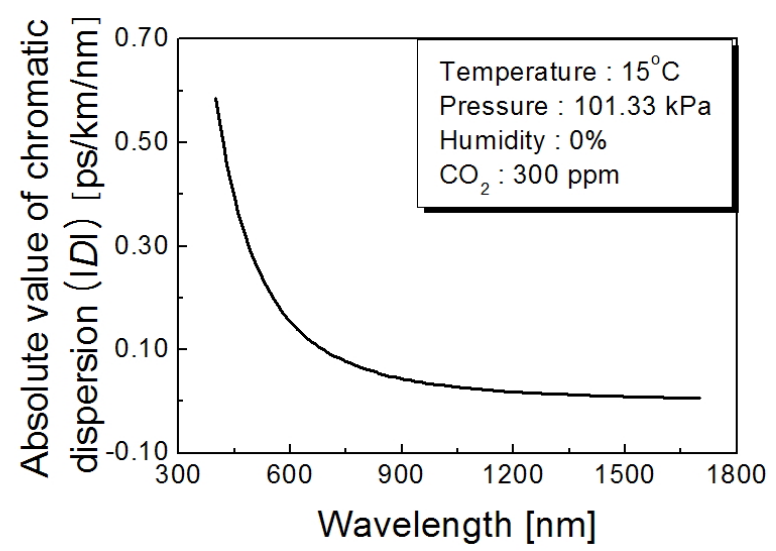

(a)
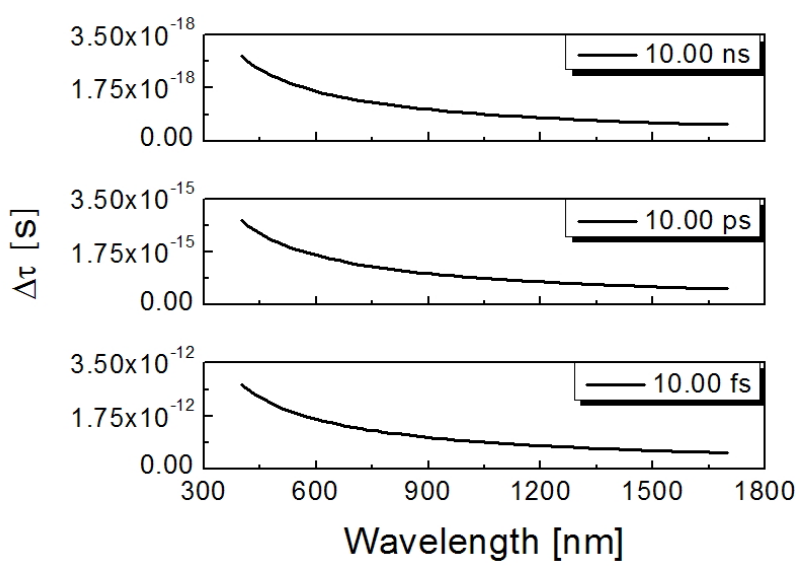

(b)

FIG. 2. (a) Absolute value of the chromatic dispersion $(|D|)$ of the standard atmosphere and (b) variation of the amount of pulse width broadening $(\Delta \tau)$ caused by the chromatic dispersion depending on the input pulse width $\left(\tau_{0}\right)$ after the 200-m round trip to the target.
TABLE 1. The amount of pulse width broadening for various pulse lasers with different initial pulse width after the 200-m round trip to the target at the operating wavelength of $1550 \mathrm{~nm}$

\begin{tabular}{c|c|c}
\hline $\begin{array}{c}\text { Types of pulse } \\
\text { laser }\end{array}$ & $\begin{array}{c}\text { Initial pulse } \\
\text { width }\end{array}$ & $\begin{array}{c}\text { Pulse broadening after the } \\
\text { 200-m round trip to the target }\end{array}$ \\
\hline ns laser & $10 \mathrm{~ns}$ & $5.90 \times 10^{-19} \mathrm{~s}$ \\
\hline ps laser & $10 \mathrm{ps}$ & $5.90 \times 10^{-16} \mathrm{~s}$ \\
\hline fs laser & $10 \mathrm{fs}$ & $5.98 \times 10^{-13} \mathrm{~s}$ \\
\hline
\end{tabular}

system should be essentially affected by nonlinear refractive index depending on the optical intensity of the laser pulse [21]. The optical intensity of pulse laser required in the TOF-LRF strongly depends on a round trip distance [6, 22]. For the 200-m round trip distance, however, high power is not necessary to the pulse laser because of a relatively short distance of the round trip, and the effect of nonlinear refractive index of air on the measurement error of the round trip distance will be negligible.

A laser pulse at a certain wavelength is generally absorbed by various molecules in air. Particularly, the water vapor and the $\mathrm{CO}_{2}$ in air are strong factors for variation in the transmission of the laser pulse propagating in air [15]. The transmission of the standard atmosphere $\left(T_{w}\right)$ with respect to the water vapor $\left(P_{w}\right)$ can be written as [17],

$$
\begin{aligned}
& T_{w}=1-\frac{2}{\sqrt{\pi}} \int_{0}^{\frac{\alpha(\lambda)}{2} \sqrt{\pi w_{w}}} \exp \left[-t^{2}\right] d t, \\
& w_{w}=2 \frac{m_{w} p_{w} D_{t}}{\rho_{l} k T}, \\
& p_{w}=2.408 \times 10^{8} h\left(\frac{300}{T+273.15}\right)^{4} \exp \left[-22.64 \times \frac{300}{T+273.15}\right],
\end{aligned}
$$

where $\alpha(\lambda)$ is an absorption coefficient of the water vapor as a function of the wavelength. $w_{w}$ is a depth of precipitable water which is defined as the depth of water in a column of the atmosphere when if all the water vapor in the optical path is condensed into a layer of liquid water [23]. $m_{w}$ is the molecular weight of water, $\rho_{l}$ is the mass density of liquid water, $k$ is the Boltzmann constant, and $T$ is the temperature. $p_{w}$ is the partial pressure of the water vapor which is closely related to the relative humidity $(h)$ [24]. By using different values of the absorption coefficient $(\alpha(\lambda))$ depending on the operating wavelength of the pulse laser, the transmission of the standard atmosphere can be calculated [16]. Figure 3(a) shows the theoretical result of transmission of the standard atmosphere $\left(T_{w}\right)$ with variations in the relative humidity for the 200-m round trip distance of femtosecond laser through air. The transmission of the standard atmosphere is changed by the relative humidity of air because of the water vapor absorption. From Eq. (14) to (16), it is evident 


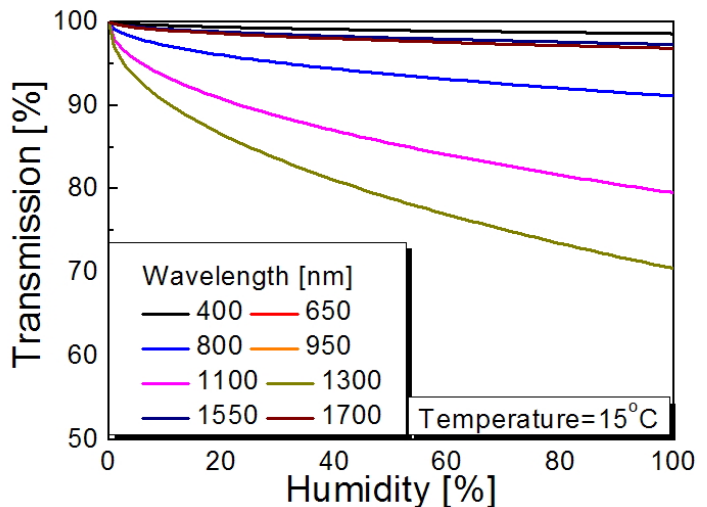

(a)

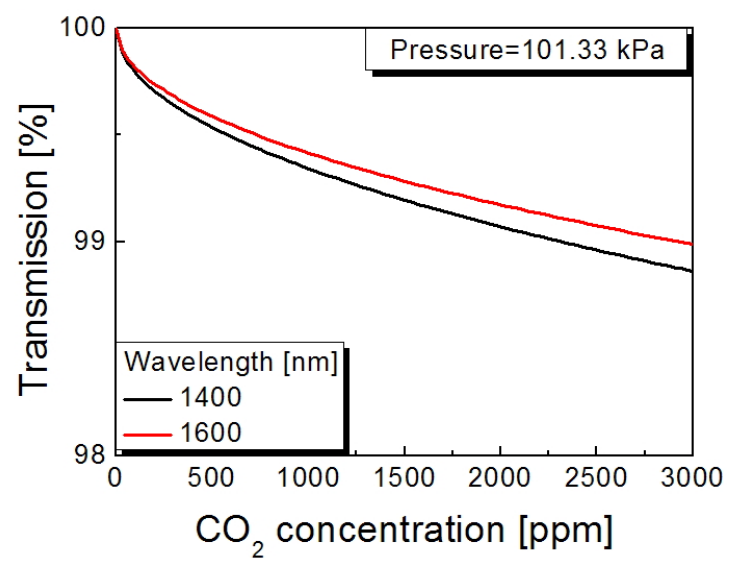

(b)

FIG. 3. Theoretical results for variation of the transmission of the standard atmosphere with respect to the relative humidity (a) and the concentration of $\mathrm{CO}_{2}$ (b).

that the transmission of the standard atmosphere depends on the partial pressure of the water vapor $\left(p_{w}\right)$, which is directly proportional to the relative humidity. Consequently, increasing the relative humidity gradually diminishes the transmission of the standard atmosphere as seen in Fig. 3(a). The transmission of the standard atmosphere corresponding to the relative humidity is also changed by the operating wavelength of the pulse laser because of the strong dependence of the absorption coefficient $(\alpha(\lambda))$ of the water vapor on the operating wavelength. The different variation of the transmission in Fig. 3(a) is caused by the different values of the absorption coefficients corresponding to the operating wavelength [16]. The transmission variation of the standard atmosphere with respect to the water vapor at an operating wavelength of $1300 \mathrm{~nm}$ is relatively higher than those at other wavelengths because of the high absorption coefficient of water vapor at $1300 \mathrm{~nm}$.

For the concentration of $\mathrm{CO}_{2}$, the transmission of the standard atmosphere $\left(T_{c}\right)$ can be written as [15],

$$
T_{c}=1-\beta(\lambda) \sqrt{2 D_{t} P x_{c}}\left(P+P x_{c}\right)^{\gamma(\lambda)},
$$

where $\beta(\lambda)$ and $\chi(\lambda)$ are the empirical parameters which are closely related to the line strength in the spectra line induced by molecular collisions based on $\mathrm{CO}_{2}$ [15]. Figure 3(b) shows the transmission variation of the standard atmosphere $\left(T_{c}\right)$ with variations in the concentration of $\mathrm{CO}_{2} \cdot \mathrm{CO}_{2}$ absorbs the optical power of the pulse laser resulting in the variation of the transmission of the standard atmosphere for the 200-m round trip distance of femtosecond laser through air. We considered two operating wavelengths with weak absorption bands of $\mathrm{CO}_{2}$, such as $1400 \mathrm{~nm}$ and $1600 \mathrm{~nm}$ [15]. By considering Eq. (17), it is readily understandable that the transmission of the standard atmosphere is reduced by increasing the concentration of $\mathrm{CO}_{2}$ as shown in Fig. 3(b). Since the absorption coefficients at $1400 \mathrm{~nm}$ is higher than those at $1600 \mathrm{~nm}$, the transmission reduction at $1400 \mathrm{~nm}$ is higher than that at $1600 \mathrm{~nm}$. By comparing the influence of water vapor and the concentration of $\mathrm{CO}_{2}$ on the transmission of the standard atmosphere, it can be seen that the absorption of water vapor is the more dominant factor in reducing the transmission.

\section{CONCLUSION}

We have analyzed the propagation characteristics of the pulse laser of TOF-LRF system with variations in atmospheric conditions, such as temperature, pressure, relative humidity, and the concentration of $\mathrm{CO}_{2}$. The refractive index variation of air induced by the temperature, pressure, relative humidity, and the concentration of $\mathrm{CO}_{2}$ results in a maximum distance measurement error of $6.42 \mathrm{~mm}, 12.01 \mathrm{~mm}, 56.86 \mu \mathrm{m}$ and $48.98 \mu \mathrm{m}$, respectively, when the laser pulse propagates the 200-m round trip distance through air. Therefore, for the realization of the precise TOF-LRF system using the femtosecond laser, the measurement error of distance measurement error with variations in the atmospheric conditions should be corrected. We also have shown the dependence of the propagation characteristics of the pulse laser on the operating wavelength. Since the air is a dispersive medium, the pulse width should be broadened because of the chromatic dispersion of the standard atmosphere when a pulse laser propagates through the air. Considering the initial pulse width, the amount of pulse width broadening of femtosecond laser is higher than that of the nanosecond laser or picosecond laser. The absolute value of the chromatic dispersion of the standard atmosphere is diminished by increasing the operating wavelength of the pulse laser. Increasing the relative humidity gradually degrades the transmission of the standard atmosphere. The transmission of the standard atmosphere corresponding to the relative humidity is also changed by the operating wavelength of the pulse laser because of the strong dependence of the absorption coefficient $(\alpha(\lambda))$ of the water vapor on the operating wavelength. The transmission of the standard atmosphere is also reduced gradually by increasing the concentration of $\mathrm{CO}_{2}$ because of the enhancement of optical absorption of $\mathrm{CO}_{2}$. 


\section{ACKNOWLEDGMENT}

This work was supported by the Basic Science Research Program through the National Research Foundation of Korea (NRF), funded by the Ministry of Education, Science and Technology (2012R1A1A2000999).

\section{REFERENCES}

1. M.-C. Amann, T. Bosch, M. Lescure, R. Myllylä, and M. Rioux, "Laser ranging: A critical review of usual techniques for distance measurement," Opt. Eng. 40, 10-19 (2001).

2. M. Laurenzis, F. Christnacher, and D. Monnin, "Long-range three-dimensional active imaging with superresolution depth mapping," Opt. Lett. 32, 3146-3148 (2007).

3. H. Ailisto, V. Heikkinen, R. Mitikka, R. Myllylä, J. Kostamovaara, A. Màntyniemi, and M. Koskinen, "Scannerless imaging pulsed-laser range finding," J. Opt. 4, S337-S346 (2002).

4. A. Biernat and G. Kompa, "Powerful picosecond laser pulses enabling high-resolution pulsed laser radar," J. Opt. 29, 225-228 (1998).

5. L. A. Vazquez-Zuniga and Y. Jeong, "Power-scalable, subnanosecond mode-locked erbium-doped fiber laser based on a frequency-shifted-feedback ring cavity incorporating a narrow bandpass filter," J. Opt. Soc. Korea 17, 117-181 (2013).

6. J. Lee, Y.-J. Kim, K. Lee, S. Lee, and S.-W. Kim, "Time-of-flight measurement with femtosecond light pulses," Nat. Photon. 4, 716-720 (2010).

7. S. H. Lee, J. H. Lee, Y. J. Kim, K. W. Lee, and S. W. $\mathrm{Kim}$, "Active compensation of large dispersion of femtosecond pulses for precision laser ranging," Opt. Express 19, 40024008 (2011).

8. P. Jian, O. Pinel, C. Fabre, B. Lamine, and N. Treps, "Real-time displacement measurement immune from atmospheric parameters using optical frequency combs," Opt. Express 20, 27133-27146 (2012).

9. H.-S. Jeong, D. W. Kim, K. H. Kim, and J.-M. Lee, "All-optical signal-conversion efficiency with a parameterdependent four-wave-mixing process in a silicon nanowaveguide," J. Korean Phys. Soc. 62, 428-434 (2013).
10. D. H. Song, S. I. Hwang, and D.-K. Ko, "Dynamics of sub-microjoule femtosecond pulse formation in a negative dispersion regime," J. Korean Phys. Soc. 61, $730-734$ (2012).

11. M. Hebert and E. Krotkov, "3D measurements from imaging laser radars: How good are they?," Img. Vision Comput. 10, 170-178 (1992).

12. B. Edlén, "The refractive index of air," Metrol. 2, 71-80 (1966).

13. P. E. Ciddor, "Refractive index of air: New equations for the visible and near infrared," Appl. Opt. 35, 1566-1573 (1996).

14. J. N. Howard, D. E. Burch, and D. Williams, "Infrared transmission of synthetic atmospheres. I. Instrumentation," J. Opt. Soc. Am. 46, 186-190 (1956).

15. J. N. Howard, D. E. Burch, and D. Williams, "Infrared transmission of synthetic atmospheres. II. Absorption by carbon dioxide," J. Opt. Soc. Am. 46, 237-241 (1956).

16. R. G. Eldridge, "Water vapor absorption of visible and near infrared radiation,” Appl. Opt. 6, 709-713 (1967).

17. H. Barrell and J. E. Sears, "The refraction and dispersion of air for the visible spectrum," Philos. Trans. R. Soc. London Ser. A Math. Phys. Sci. 238, 1-64 (1939).

18. K. P. Birch and M. J. Downs, "An updated Edlén equation for the refractive index of air," Metrologia 30, 155-162 (1993).

19. M. Tateda, N. Shibata, and S. Seikai, "Interferometric method for chromatic dispersion measurement in a single-mode optical fiber," IEEE J. Quantum Electron. 17, 404-407 (1981).

20. K. L. Sala, G. A. Kenney-Wallace, and G. E. Hall, "CW autocorrelation measurements of picosecond laser pulses," IEEE J. Quantum Electron. 16, 990-996 (1980).

21. W. E. Martin and R. J. Winfield, "Nonlinear effects on pulsed laser propagation in the atmosphere," Appl. Opt. 27, 567-577 (1988).

22. Rairoux, H. Schillinger, S. Niedermeier, M. Rodriguez, F. Ronneberger, R. Sauerbrey, B. Stein, D. Waite, C. Wedekind, H. Wille, L. Wöste, and C. Ziener, "Remote sensing of the atmosphere using ultrashort laser pulses," Appl. Phys. B 71, 573-580 (2000).

23. S. E. Tuller, "The relationship between precipitable water vapor and surface humidity in New Zealand," Arch. Met. Geoph. Biokl. 26, 197-212 (1977).

24. H. J. Liebe, "MPM-An atmospheric millimeter-wave propagation model,” Int. J. Infrared Mill. Waves 10, 631-650 (1989). 